\title{
A FUNCTIONAL IN VIVO SHRNA SCREEN FOR THE IDENTIFICATION OF TRIPLE-NEGATIVE BREAST CANCER METASTASIS SUPPRESSOR GENES
}

Rebeka Tomasin¹, Ana Carolina Baptista Moreno Martin, Márcia Regina Cominetti , Kaylene Simpson², Robin Anderson ${ }^{3}$, Richard Redvers ${ }^{3}$

${ }^{1}$ Laboratory of Biology of Aging, Department of Gerontology, Universidade federal de São Carlos - São Carlos (SP), Brazil. 2Peter MacCallum Cancer Centre, Victorian Comprehensive Cancer Centre - Australia.

${ }^{3}$ Olivia Newton John Cancer Research Institute - Australia.

Objective: When diagnosed early, breast cancer can be treated with a great rate of success. However, once the disease reaches metastatic stage, spreading to organs such as lungs and bones, the available treatments are extremely limited and prognosis is poor. This is particularly true for the triple-negative subtype. By identifying genes that regulate metastasis, it would be possible to predict the patients whose disease is more likely to spread, and it would create an opportunity to develop more effective therapies. Methods: Using a genome-wide lentiviral shRNA library, we initiated an in vivo functional screen aiming to identify genes whose reduction in expression levels, in non/poorly metastatic murine triple-negative breast cancer cells (67NRch1/66cl4ch14, respectively), would lead to spontaneous metastasis in vivo. Results: Dozens of putative metastasis suppressors were identified, in a wide range of organs, by conventional and next generation sequencing. These candidates were further subjected to an in vitro RNAi screen in 66cl4ch14 cells, involving viability, wound healing and adhesion assays. Conclusion: Integrative analysis of both screens (in vivo and in vitro), literature review, RNA-seq and bioinformatics analysis of gene expression and clinical outcome (Oncomine/ Breastmark) indicated highly-confidence candidates to be taken for further analysis and validation in vivo. Additional in vivo and in vitro experiments, aiming to validate the obtained candidates as bona fide metastasis suppressor genes, as well as elucidate their possible mechanisms of action, are ongoing. 